\title{
Accounting for Missing Data in Clinical Research
}

\author{
John R. Stevens, PhD, \\ Department of Mathematics and Statistics, Utah State University, Logan, Utah
}

Anvar Suyundikov, PhD, and

BioStat Solutions Inc., Frederick, Maryland

Martha L. Slattery, PhD, MPH

Department of Internal Medicine, University of Utah School of Medicine, Salt Lake City, Utah

\section{To the Editor}

Drs Newgard and Lewis ${ }^{1}$ provided an overview of many important issues to consider when dealing with missing data in clinical research. In particular, they summarized the limitations of traditional imputation methods - failure to account for uncertainty in imputed values, failure to make full use of observed values, possibilities for bias, and artificially low variance.

One imputation method in use for many years involves the K-Nearest Neighbors (KNN) approach, ${ }^{2}$ which was not included in the JAMA Guide to Statistics and Methods article. In the KNN method, a variable's missing value for a participant is imputed by taking a weighted average of fully observed variable values for several participants that are the most similar (in terms of other covariates) to the person in question. KNN has been widely applied with considerable effectiveness, including in "-omics" clinical research. ${ }^{3}$

At the same time, KNN is one of the methods that "replaces a missing value with a single number ... and can threaten the validity of study results." Newgard and Lewis may have excluded KNN from their review for this reason, leading to their concluding statement that "In general, multiple imputation is the best approach for modeling the effects of missing data in studies."

However, a recent modification ${ }^{4}$ to the KNN method efficiently resolves these limitations, as demonstrated in a colorectal cancer study. This modification quantifies the dependence induced by imputation, particularly between imputed values and the fully observed values on which they are based. This dependence can be accounted for in subsequent statistical tests, effectively accounting for uncertainty (or chance variability) in the imputed values, making full use of observed values, avoiding systematic bias, and appropriately adjusting variance estimates.

Corresponding Author: John R. Stevens, PhD, Department of Mathematics and Statistics, Utah State University, 3900 Old Main Hill, Logan, UT 84322-3900 (john.r.stevens@usu.edu).

Conflict of Interest Disclosures: The authors have completed and submitted the ICMJE Form for Disclosure of Potential Conflicts of Interest and reported receiving a grant to the University of Utah from the National Cancer Institute. 
Using this approach has been shown to achieve greater accuracy than the multiple imputation method, as well as better sensitivity than doing no imputation at all. Compared to this KNN-dependent method, the multiple imputation method preferred by Newgard and Lewis does have greater sensitivity, but also lower specificity (and in fact can fail to control the type I error rate entirely). ${ }^{4}$

As data collection methods in clinical research become more comprehensive, the nature of missing data will continue to evolve, and statistical methods for missing data will need to be updated accordingly.

\section{References}

1. Newgard CD, Lewis RL. Missing data: How to best account for what is not known. JAMA. 2015; 314(9):940-941. [PubMed: 26325562]

2. Cover TM, Hart PE. Nearest neighbor pattern classification. IEEE Transactions on Information Theory. 1967; 13(1):21-27.

3. Troyanskaya O, Cantor M, Sherlock G, et al. Missing value estimation methods for DNA microarrays. Bioinformatics. 2001; 17(6):520-525. [PubMed: 11395428]

4. Suyundikov A, Stevens JR, Corcoran C, Herrick J, Wolff RK, Slattery ML. Accounting for dependence induced by weighted KNN imputation in paired samples, motivated by a colorectal cancer study. PLOS ONE. 2015; 10(4):e0119876. [PubMed: 25849489] 